\title{
Nonlinear Green's Functions for Wave Equation with Quadratic and Hyperbolic Potentials
}

\author{
Asatur Zh. Khurshudyan \\ Department of Dynamics of Deformable Systems and Coupled Fields, Institute of Mechanics, National Academy of Sciences of Armenia, \\ 24B Baghramyan Ave., 0019 Yerevan, Armenia
}

Correspondence should be addressed to Asatur Zh. Khurshudyan; khurshudyan@mechins.sci.am

Received 2 February 2018; Revised 7 March 2018; Accepted 29 April 2018; Published 3 June 2018

Academic Editor: Kaliyaperumal Nakkeeran

Copyright (C) 2018 Asatur Zh. Khurshudyan. This is an open access article distributed under the Creative Commons Attribution License, which permits unrestricted use, distribution, and reproduction in any medium, provided the original work is properly cited.

\begin{abstract}
The advantageous Green's function method that originally has been developed for nonhomogeneous linear equations has been recently extended to nonlinear equations by Frasca. This article is devoted to rigorous and numerical analysis of some secondorder differential equations new nonlinearities by means of Frasca's method. More specifically, we consider one-dimensional wave equation with quadratic and hyperbolic nonlinearities. The case of exponential nonlinearity has been reported earlier. Using the method of generalized separation of variables, it is shown that a hierarchy of nonlinear wave equations can be reduced to secondorder nonlinear ordinary differential equations, to which Frasca's method is applicable. Numerical error analysis in both cases of nonlinearity is carried out for various source functions supporting the advantage of the method.
\end{abstract}

\section{Introduction}

The most simplistic models for real-life objects and phenomena are formulated in terms of linear constraints. For a better understanding of various phenomena having a nonlinear character, strongly nonlinear constraints must be involved. Nevertheless, numerical and especially rigorous analysis of nonlinear constraints can be significantly sophisticated and can require burdensome computational costs. In such cases, the so-called semianalytical methods such as Adomian decomposition method [1], Hirota direct method [2], and $\left(G^{\prime} / G\right)$ expansion method [3] usually become very convenient. The reason is that they allow deriving an approximate analytical solution to the nonlinear constraints and, on its basis, to perform a sensitivity analysis of the solution dependence on, e.g., boundary/initial data and external influence.

For linear constraints, the sensitivity analysis is usually carried out by Green's function method [4]. The solution of nonhomogeneous differential equations is represented in the form of convolution of Green's function and the righthand side of the equation. At this, Green's representation formula is derived on the basis of the superposition principle. Therefore it holds true exceptionally for linear constraints. Nevertheless, there exist several studies attempting to generalize Green's function concept and Green's representation formula to nonlinear systems. An exact extension has been reported in [5-7] (see also other works by Cacuci). Assuming that the first Gatoux derivative of the state equation exists, the concept of the forward and backward propagators is introduced and the general solution is represented as the inner product of the propagators with the right-hand side (nonlinear Green's representation formula). In other words, the propagators play the same role for nonlinear systems as Green's function does for linear systems.

Another extension has been reported a decade ago by Frasca in $[8,9]$. It has been shown that the general solution of the nonlinear "oscillating" equations of the form

$$
\frac{d^{2} w}{d t^{2}}+N(w, t)=f(t), \quad t>0
$$


for a generic nonlinear function $N$, a given right-hand side $f$, and appropriate Cauchy conditions can be represented in terms of the short time expansion as follows:

$$
\begin{aligned}
w(t)= & a_{0} \int_{0}^{t} G(t-\tau) f(\tau) \mathrm{d} \tau \\
& +a_{1} \int_{0}^{t}(t-\tau) G(t-\tau) f(\tau) \mathrm{d} \tau \\
& +a_{2} \int_{0}^{t}(t-\tau)^{2} G(t-\tau) f(\tau) \mathrm{d} \tau \\
& +a_{3} \int_{0}^{t}(t-\tau)^{3} G(t-\tau) f(\tau) \mathrm{d} \tau+\cdots \\
= & \sum_{k=0}^{\infty} a_{k} \int_{0}^{t}(t-\tau)^{k} G(t-\tau) f(\tau) \mathrm{d} \tau
\end{aligned}
$$

where $a_{k}, k=0,1,2, \ldots$, are the unknown expansion coefficients determined in terms of the quantities $w^{(k)}(0)$. Usually, in numerical computations, a truncated part of expansion (2) is considered once the required precision is achieved. In [10] it has been shown that if the consideration is restricted to the interval $t \in[0,1]$, the first-order term

$$
w(t) \approx a_{0} \int_{0}^{t} G(t-\tau) f(\tau) \mathrm{d} \tau
$$

provides an efficient approximation.

Above, $G$ is the formal extension of Green's function, i.e., the general solution of the corresponding nonlinear differential equation

$$
\frac{d^{2} G}{d t^{2}}+N(G, t)=s \delta(t), \quad t>0
$$

under appropriate Cauchy conditions; $\delta$ is the Dirac function

$$
\delta(t)= \begin{cases}0, & t \neq 0 \\ \infty, & t=0\end{cases}
$$

and $s$ is a scale factor for minimizing the approximation error. It is noteworthy that in Frasca's original paper $a_{0}=1$. It was introduced in [10] to reduce the approximation error further. Moreover, for the comparison of the short time expansion with the functional iteration method, see [9].

Here we study new forms of $N$, for which nonlinear Green's function is determined from (4) exactly. More specifically, we consider the cases of quadratic and hyperbolic nonlinearities (see Section 3). Moreover, we show that there exists a hierarchy of nonlinear wave equations that can be reduced to a second-order nonlinear differential equations, the solution of which can be represented by nonlinear Green's formula (2). For the sake of simplicity, in this paper we restrict ourselves only by the first-order approximation (3). A numerical error analysis (see Section 4) is carried out in comparison with the well-known method of lines (MoL). It is established that even the first-order approximation gives a solution compatible with a numerical one. The approximation error can be reduced further by considering higher-order terms in the short time expansion above.

Such techniques are quite useful for a deeper practical analysis, since they allow avoidance of any linearization of the state equation, which often leads to a loss of some key information about the nature of the nonlinear processes [11, 12]. Note that this approach is applicable as long as nonlinear Green's equation (4) is resolvable under the corresponding Cauchy conditions. Note also that the idea of $[13,14]$ can be applied on the results of this paper, in order to consider control problems for new nonlinear dynamic systems described by the "oscillating" equations (1) and related partial differential equations.

\section{Exactly Integrable Cases}

Two particular nonlinearities are considered in [8] allowing construction of the exact solution of (4). Particularly, the cubic nonlinearity

$$
N(w, t)=w^{3}
$$

provides

$$
G(t)=2^{1 / 4} \theta(t) \cdot \operatorname{sn}\left[\frac{t}{2^{1 / 4}}, i\right],
$$

where $\theta$ is the Heaviside function

$$
\theta(t)= \begin{cases}1, & t>0, \\ \frac{1}{2}, & t=0, \\ 0, & t<0,\end{cases}
$$

and sn is the Jacobi snoidal function. Here it is taken into account that in the sense of distributions

$$
\theta^{\prime}(t)=\delta(t)
$$

Furthermore, the trigonometric nonlinearity

$$
N(w, t)=\sin w
$$

admits exact integration of (4) as follows:

$$
G(t)=2 \theta(t) \cdot \operatorname{am}\left[\frac{t}{\sqrt{2}}, \sqrt{2}\right] .
$$

Here am is the Jacobi amplitude function.

Some new nonlinearities are studied in [10]. In particular, the reciprocal and exponential nonlinearities are shown to be exactly integrable cases. For instance, in the case of the exponential nonlinearity (Liouville equation)

$$
N(w, t)=\exp w,
$$

nonlinear Green's function is found as follows:

$$
G(t)=\theta(t) \cdot \ln \left[1-\tanh ^{2}\left(\frac{t}{\sqrt{2}}\right)\right] .
$$




\section{Generalized Variable Separation and Wave Equations with Nonlinear Potentials}

Consider the one-dimensional wave equation

$$
\begin{aligned}
\frac{\partial^{2} \widetilde{w}}{\partial t^{2}}=\frac{\partial}{\partial x}\left[\exp (\lambda x) \frac{\partial \widetilde{w}}{\partial x}\right]+\widetilde{N}(\widetilde{w}, x, t), & \\
& -\infty<x<\infty, t>0,
\end{aligned}
$$

describing the nonlinear wave propagation in inhomogeneous media with the quadratic nonlinearity

$$
\widetilde{N}(\widetilde{w}, x, t)=\widetilde{w}^{2} .
$$

The transformation $[15,16]$

$$
\chi^{2}=\exp (-2 x)-t^{2}
$$

reduces the wave equation (14) to

$$
\frac{d^{2} w}{d \chi^{2}}+w^{2}(\chi)=0
$$

where

$$
w(\chi)=\widetilde{w}(x, t) .
$$

In this case, Green's function is determined as follows:

$$
G(\chi)=-\frac{1}{c} \theta(\chi) \cdot \wp\left(c \chi+c_{1} ; 0, c_{2}\right), \quad c=(-6)^{-1 / 3},
$$

subject to appropriate Cauchy conditions. Here $\wp$ is the Weierstrass elliptic function

$$
\begin{aligned}
\wp & \left(\chi ; \omega_{1}, \omega_{2}\right) \\
= & \frac{1}{\chi^{2}} \\
& \quad+\sum_{n^{2}+m^{2} \neq 0}\left[\frac{1}{\left(\chi+\omega_{1} m+\omega_{2} n\right)^{2}}-\frac{1}{\left(\omega_{1} m+\omega_{2} n\right)^{2}}\right],
\end{aligned}
$$

and $c_{1}$ and $c_{2}$, as in all cases below, are integration constants that must be determined from the corresponding Cauchy conditions.

The same transformation reduces (14) with

$$
\widetilde{N}(\widetilde{w}, x, t)=\frac{1}{\widetilde{w}}
$$

to

$$
\frac{d^{2} w}{d \chi^{2}}+\frac{1}{w(\chi)}=0
$$

In this case, Green's function reads as

$$
G(\chi)=c_{1} \theta(\chi) \cdot \exp \left[-\varphi^{2}\left(\chi ; c_{1}, c_{2}\right)\right]
$$

where

$$
\varphi\left(\chi ; c_{1}, c_{2}\right)=\operatorname{erf}^{-1}\left[-\sqrt{\frac{2}{\pi}}\left|c_{1}\right|\left|\chi+c_{2}\right|\right]
$$

$\operatorname{erf}^{-1}$ is the inverse of the Gauss error function

$$
\operatorname{erf}(\chi)=\frac{2}{\sqrt{\pi}} \int_{0}^{\chi} \exp \left[-\xi^{2}\right] \mathrm{d} \xi
$$

Consider also the case when

$$
\widetilde{N}(\widetilde{w}, x, t)=\tanh \widetilde{w},
$$

leading in (14) to

$$
\frac{d^{2} w}{d \chi^{2}}+\tanh w(\chi)=0
$$

This case is interesting from the perspective that corresponding Green's function is found implicitly as the solution of

$$
\left[\int_{0}^{G(\chi)} \frac{1}{\sqrt{c_{1}-2 \ln (\cosh z)}} \mathrm{d} z\right]^{2}=\left(\chi+c_{2}\right)^{2} .
$$

If the solution of the ordinary differential equations above is approximated by (3), then the general solution of the wave equation (14) can be approximated by

$$
w(x, t) \approx s_{2} \int_{-\infty}^{\infty} \int_{0}^{t} \widetilde{G}(x-\xi, t-\tau) f(\xi, \tau) \mathrm{d} \xi \mathrm{d} \tau,
$$

where

$$
\widetilde{G}(x, t)=G(\chi(x, t)),
$$

and $f$ represents either boundary/initial data or the righthand side.

Nonlinear wave equations like (14) arise, e.g., in biology [17], in many areas of physics, mechanics, and engineering, describing, as a rule, nonlinear vibrations in solids or fluids [18]. In particular, they describe vibrations of a pendulum, vibrations of nonlinear elastic rods, nonlinear electromagnetic oscillations, nonlinear gravitational waves, etc.

\section{Numerical versus Green's solutions}

In this section we study the error of approximation by (3) numerically for some of the nonlinearities considered in the previous section. Various source functions are considered. The approximation error is evaluated by the logarithmic function

$$
\operatorname{Er}(t)=\log _{10}\left|w_{\text {Green's }}(t)-w_{\mathrm{MoL}}(t)\right|,
$$

measuring the absolute error between nonlinear Green's solution $w_{\text {Green's }}$ and the numerical solution $w_{\text {MoL }}$, derived by means of the method of lines, in degrees of 10 . For $w_{\text {Green's }}$ we use the first-order approximation (3).

4.1. Quadratic Potential. Let the nonlinear potential be given by (15). First, consider the case when $f(t)=\delta(t)$. Figure 1, where the discrete plot of $w_{\text {Green's }}$ and $w_{\mathrm{MoL}}$ and the logarithmic error function Er are plotted, shows an impressively small approximation error. Moreover, consideration of several other source functions such as switching, trigonometric, 

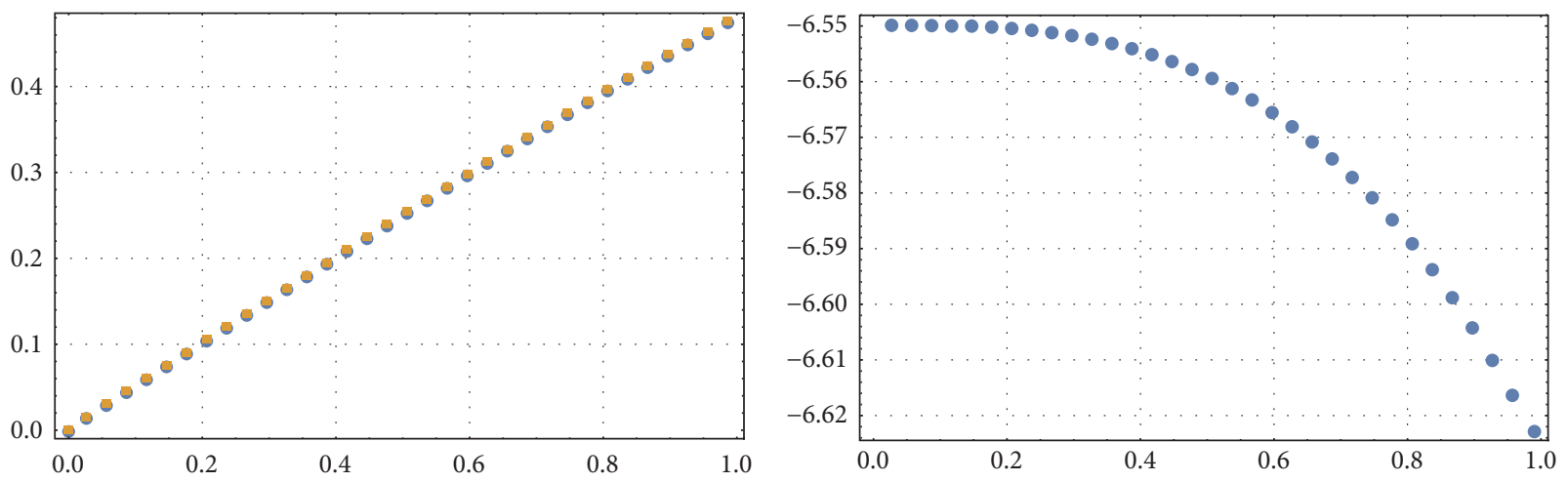

- Green's

- MoL

(a)

(b)

FIGURE 1: Discrete plot of $w_{\text {Green's }}$ and $w_{\mathrm{MoL}}$ (a) and $\operatorname{Er}(\mathrm{b})$ against $t \in[0,1]$ for $f(t)=\delta(t)$ : quadratic potential.
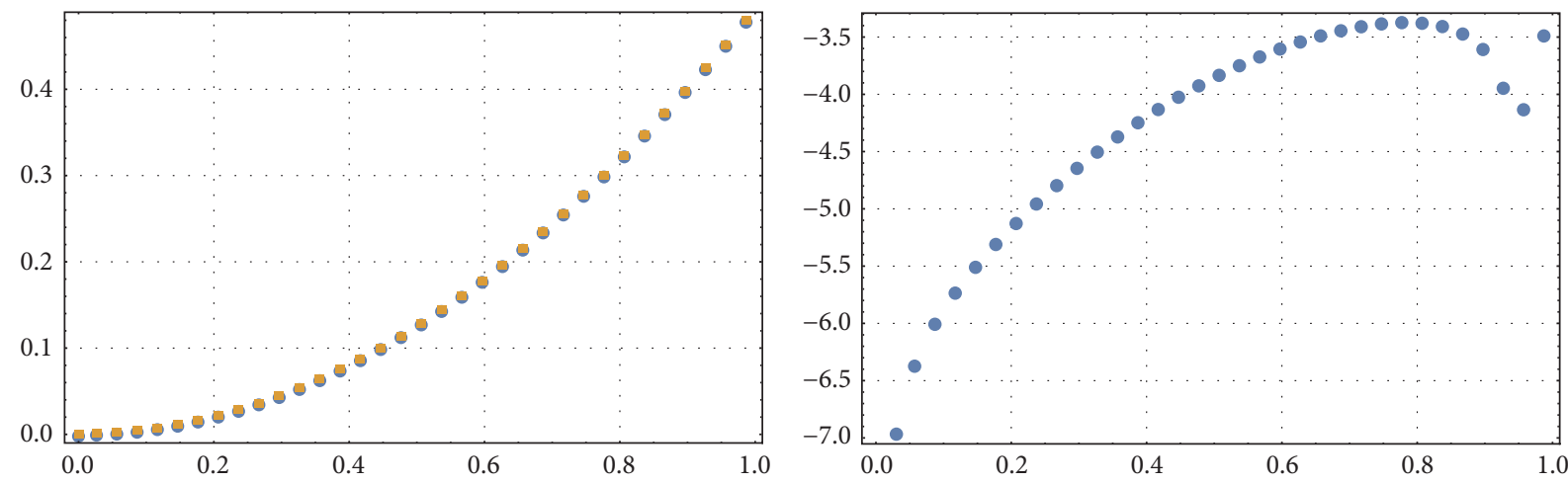

- Green's

- MoL

(a)

(b)

FIGURE 2: Discrete plot of $w_{\text {Greens }}$ and $w_{\mathrm{MoL}}$ (a) and $\operatorname{Er}(\mathrm{b})$ against $t \in[0,1]$ for $f(t)=\theta(t)$ : quadratic potential.
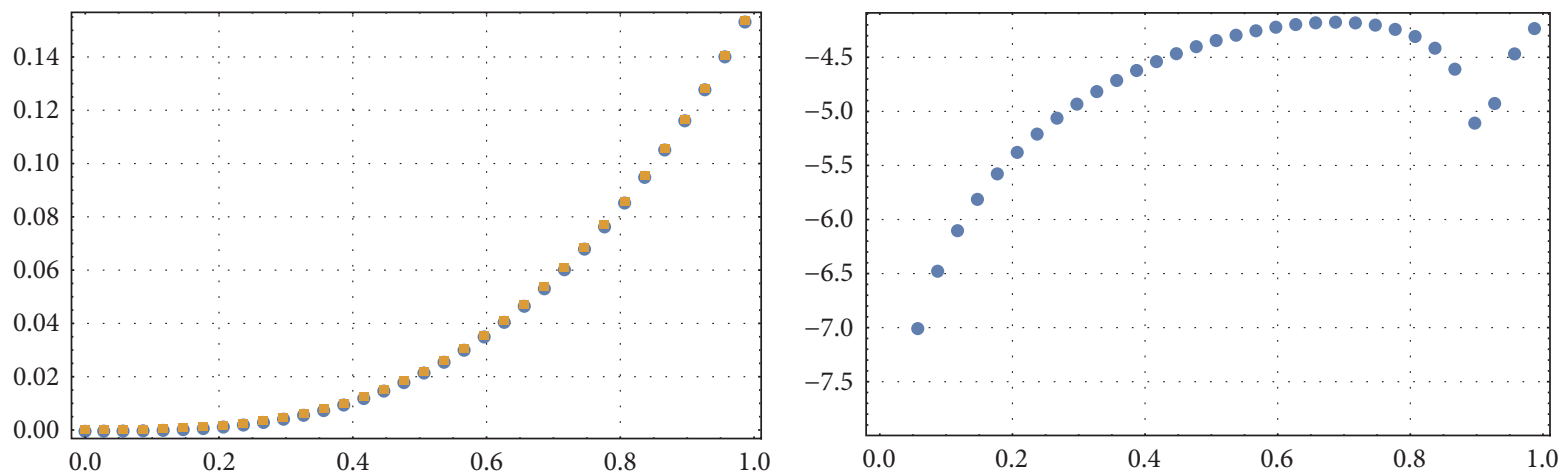

- Green's

- MoL

FIGURE 3: Discrete plot of $w_{\text {Green's }}$ and $w_{\mathrm{MoL}}$ (a) and $\operatorname{Er}(\mathrm{b})$ against $t \in[0,1]$ for $f(t)=\sin (t)$ : quadratic potential. 

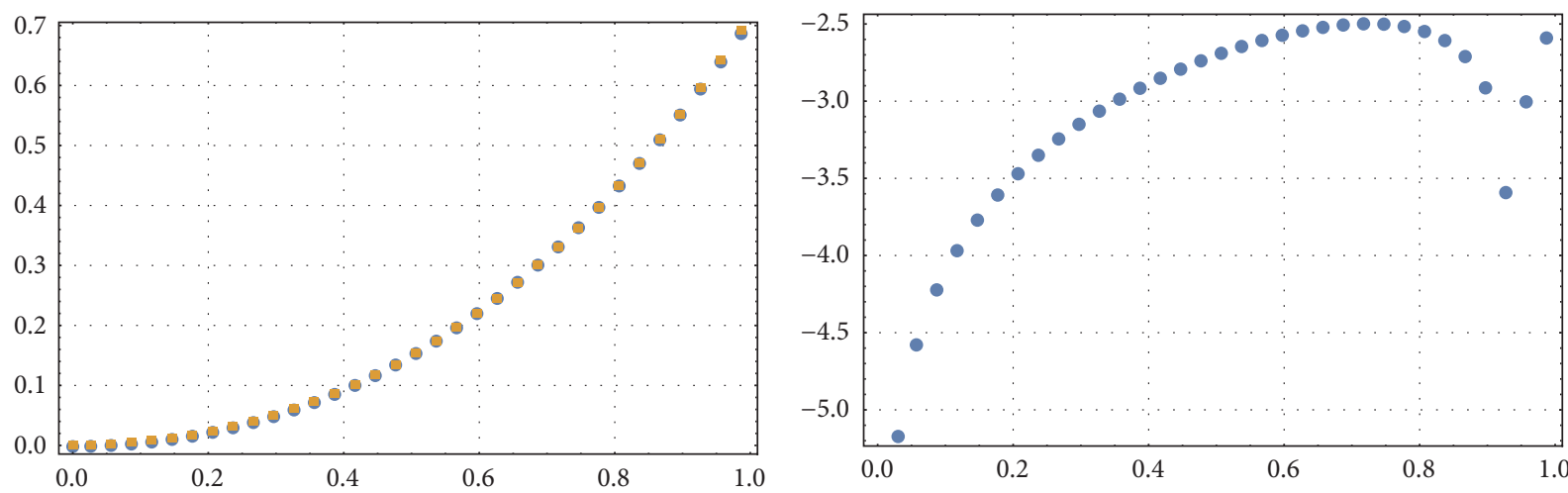

- Green's

- MoL

(a)

(b)

FIGURE 4: Discrete plot of $w_{\text {Greens }}$ and $w_{\text {MoL }}$ (a) and $\operatorname{Er}(\mathrm{b})$ against $t \in[0,1]$ for $f(t)=\exp (t)$ : quadratic potential.
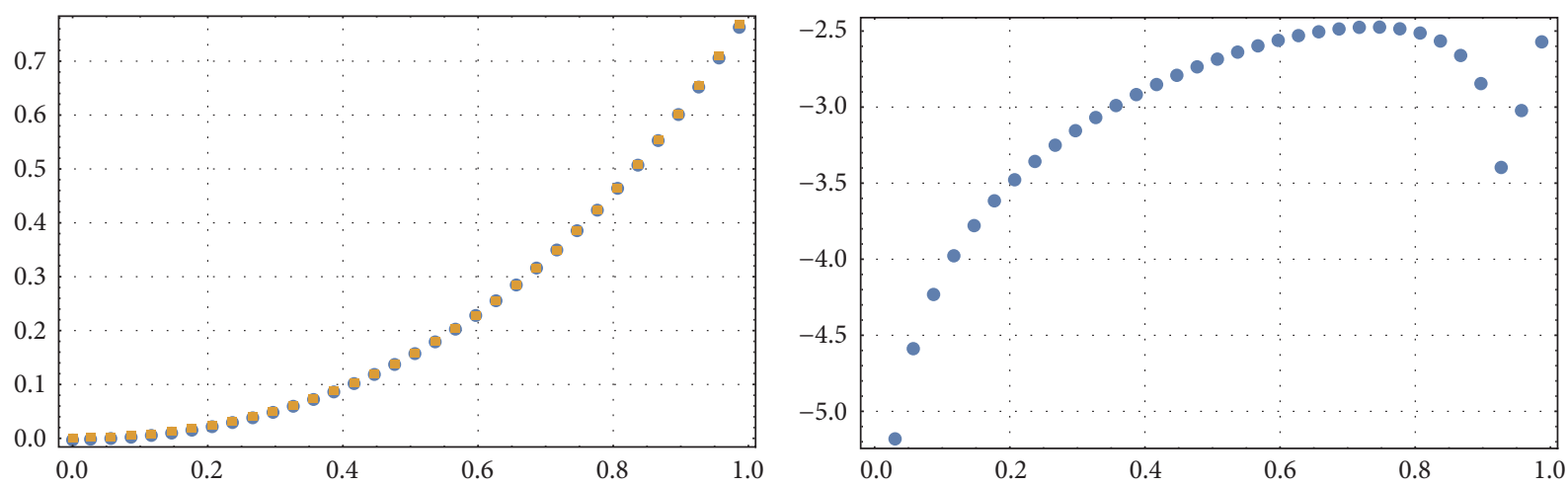

- Green's

- MoL

(a)

(b)

FIGURE 5: Discrete plot of $w_{\text {Green's }}$ and $w_{\mathrm{MoL}}$ (a) and $\operatorname{Er}(\mathrm{b})$ against $t \in[0,1]$ for $f(t)=1+t+t^{2}+t^{3}$ : quadratic potential.
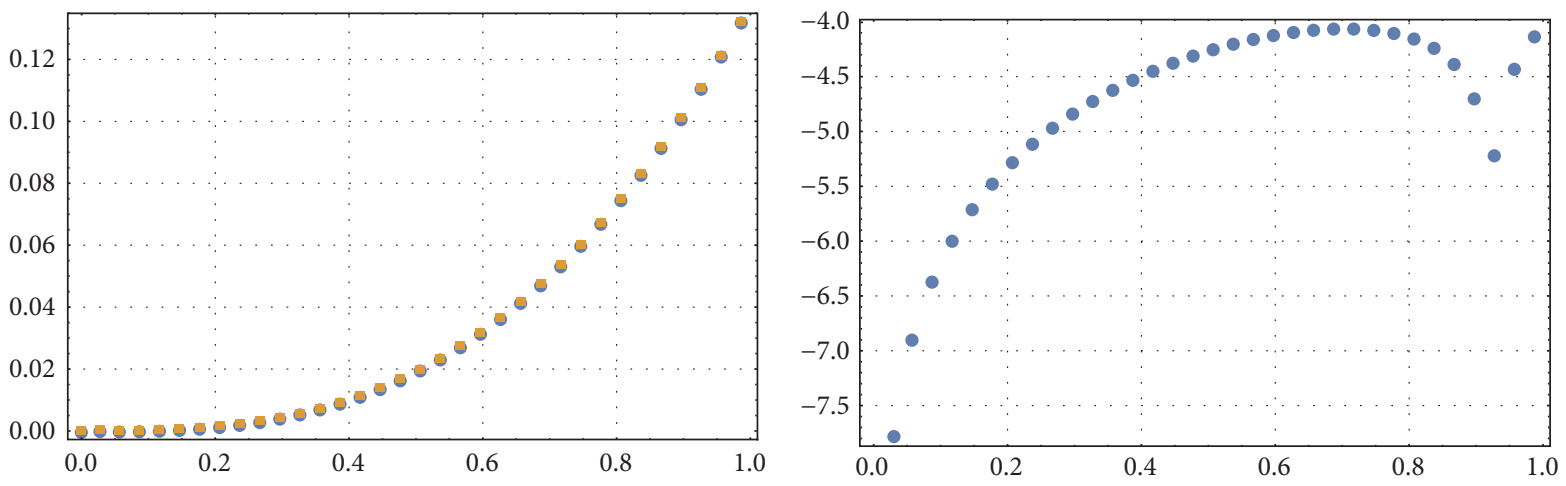

- Green's

- MoL

(a)

(b)

FIGURE 6: Discrete plot of $w_{\text {Greens }}$ and $w_{\mathrm{MoL}}$ (a) and $\operatorname{Er}(\mathrm{b})$ against $t \in[0,1]$ for $f(t)=\ln (1+t)$ : quadratic potential. 

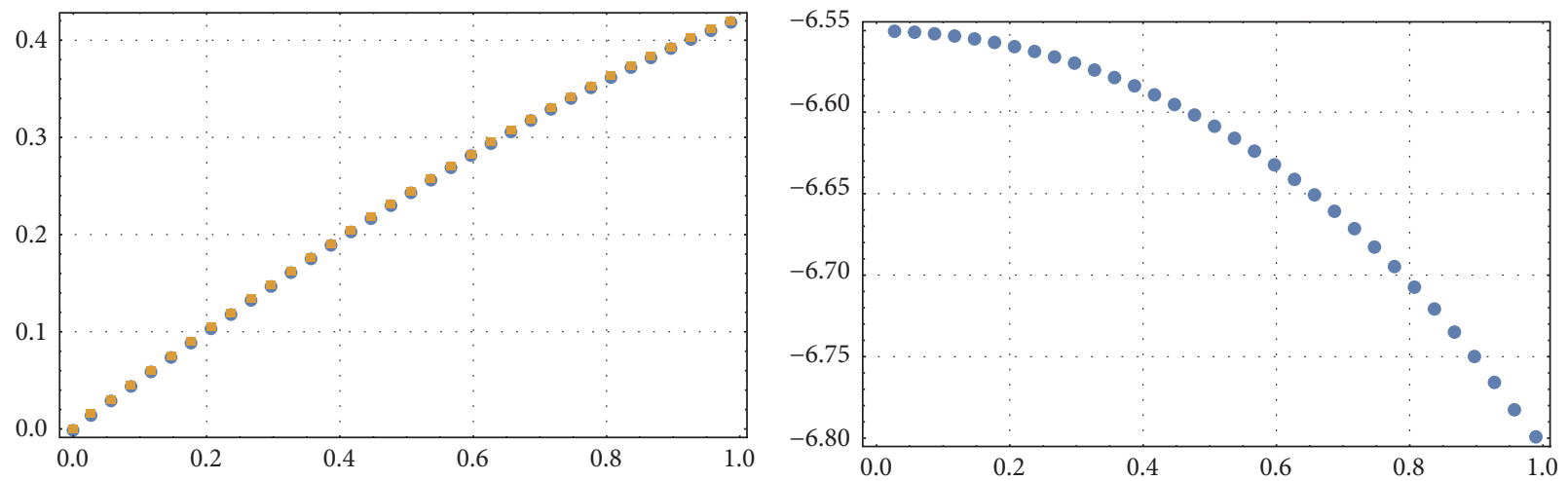

- Green's

- $\mathrm{MoL}$

(a)

(b)

FIGURE 7: Discrete plot of $w_{\text {Green's }}$ and $w_{\mathrm{MoL}}(\mathrm{a})$ and $\operatorname{Er}(\mathrm{b})$ against $t \in[0,1]$ for $f(t)=\delta(t)$ : hyperbolic potential.
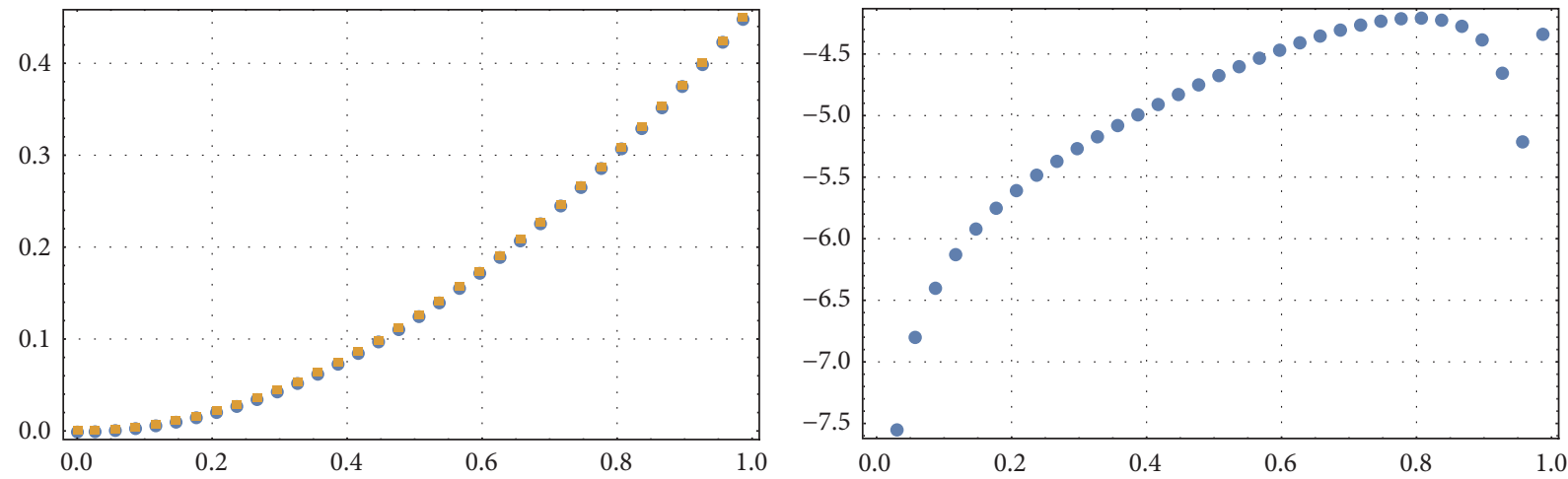

- Green's

- MoL

(a)

(b)

FIGURE 8: Discrete plot of $w_{\text {Green's }}$ and $w_{\mathrm{MoL}}$ (a) and $\operatorname{Er}(\mathrm{b})$ against $t \in[0,1]$ for $f(t)=\theta(t)$ : hyperbolic potential.
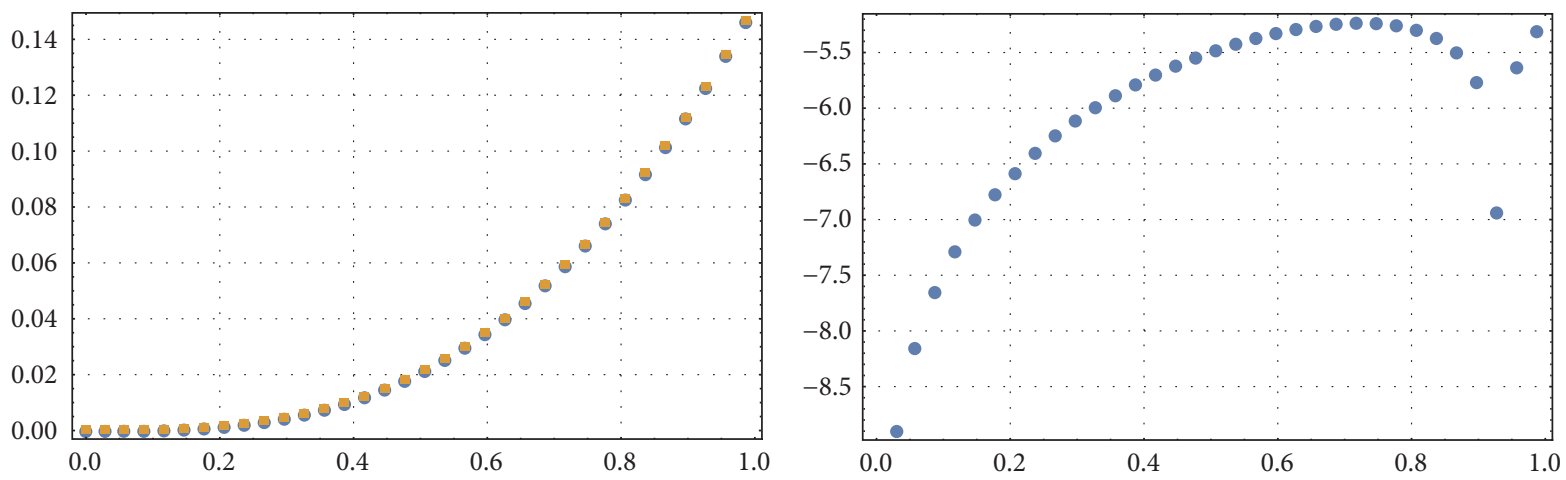

- Green's

- MoL

(a)

(b)

FIGURE 9: Discrete plot of $w_{\text {Green's }}$ and $w_{\mathrm{MoL}}$ (a) and $\operatorname{Er}(\mathrm{b})$ against $t \in[0,1]$ for $f(t)=\sin (t)$ : hyperbolic potential. 

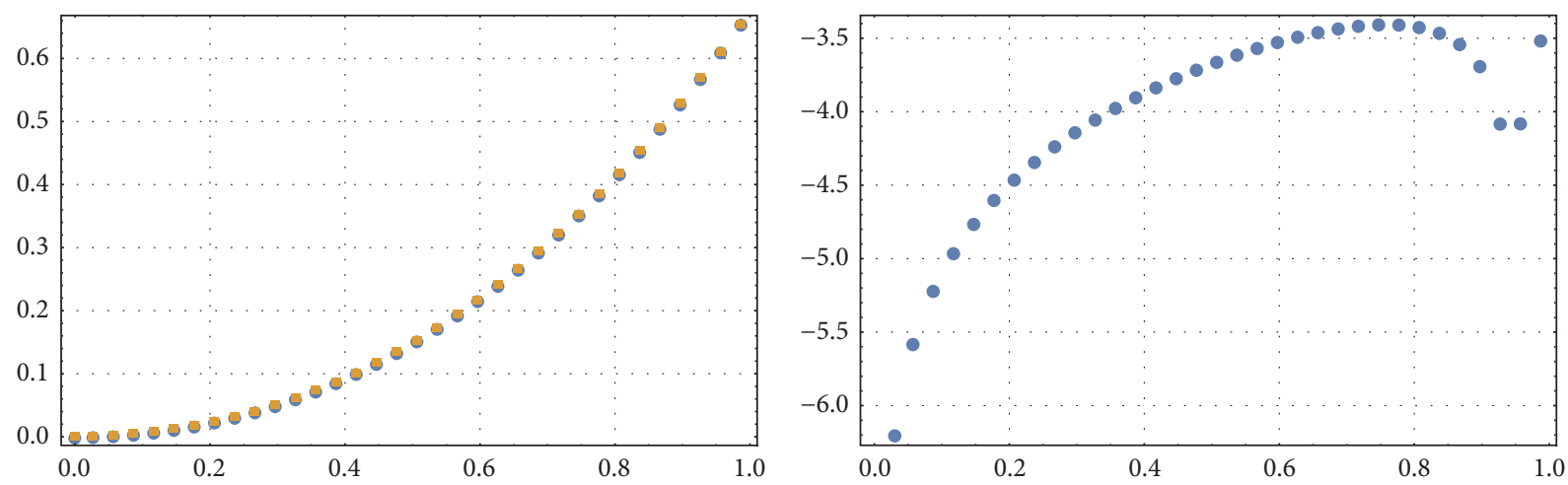

- Green's

- MoL

(a)

(b)

FIGURE 10: Discrete plot of $w_{\text {Green's }}$ and $w_{\text {MoL }}$ (a) and Er (b) against $t \in[0,1]$ for $f(t)=\exp (t)$ : hyperbolic potential.
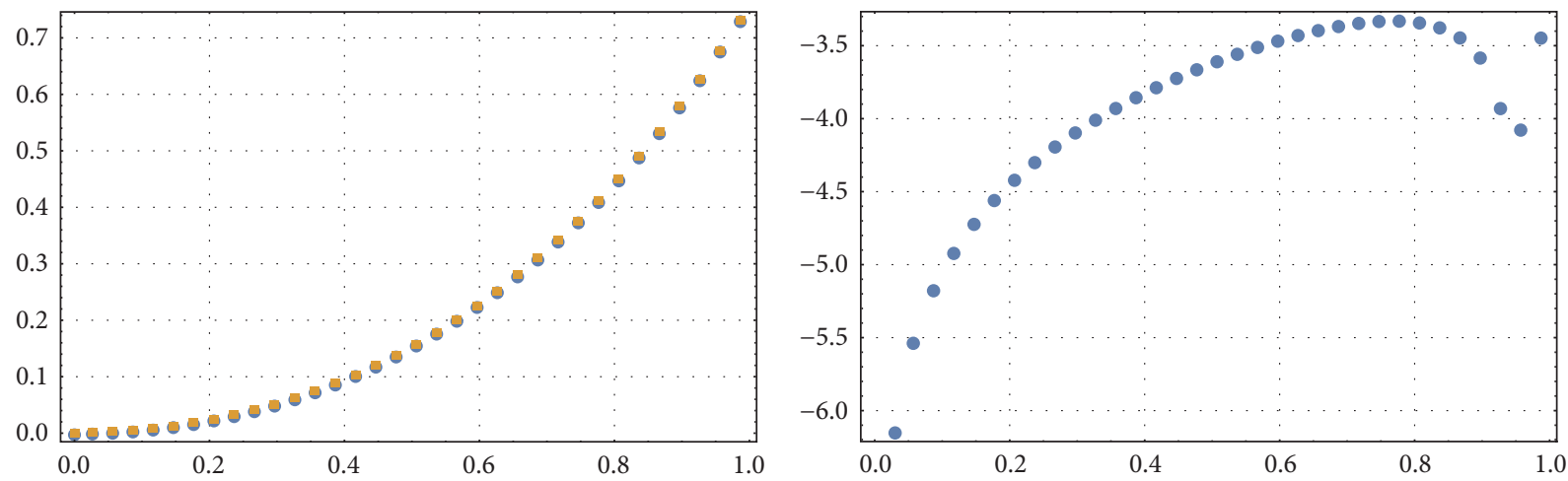

- Green's

- MoL

(a)

(b)

FIGURE 11: Discrete plot of $w_{\text {Green's }}$ and $w_{\mathrm{MoL}}$ (a) and $\operatorname{Er}(\mathrm{b})$ against $t \in[0,1]$ for $f(t)=1+t+t^{2}+t^{3}$ : hyperbolic potential.

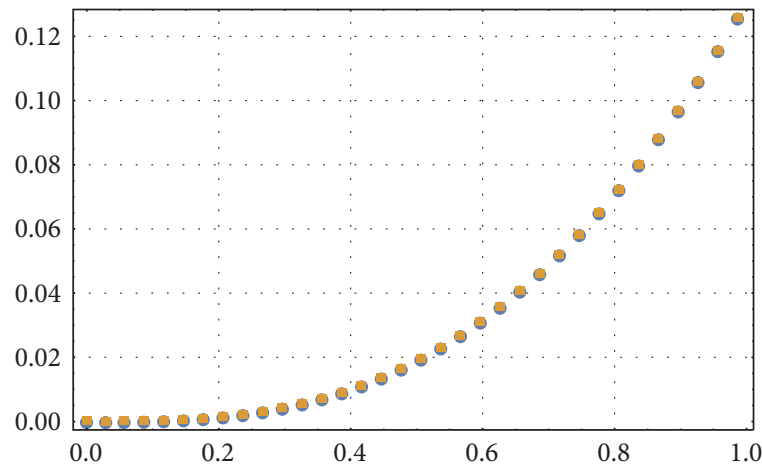

- Green's

- MoL

(a)

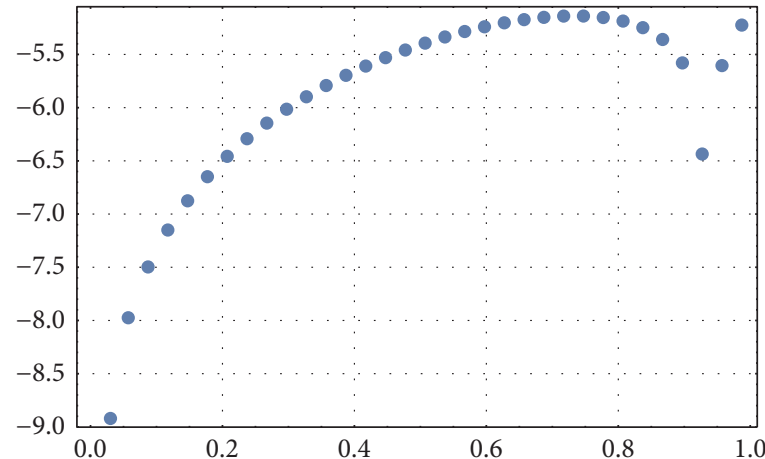

(b)

FIGURE 12: Discrete plot of $w_{\text {Green's }}$ and $w_{\text {MoL }}$ (a) and Er (b) against $t \in[0,1]$ for $f(t)=\ln (1+t)$ : hyperbolic potential. 
TABLE 1: Minimal and maximal logarithmic errors of approximation for various source functions: quadratic potential.

\begin{tabular}{lcccc}
\hline$f(t)$ & $\min \operatorname{Er}$ & $\max \operatorname{Er}$ & $a_{0}$ & $s$ \\
\hline$\delta(t)$ & -6.63 & -6.55 & 2 & 1 \\
$\theta(t)$ & -8 & -3.5 & 2.1475 & 0.93107 \\
$\sin (t)$ & -9.5 & -4.25 & 2.7807 & 0.72126 \\
$\exp (t)$ & -6 & -2.5 & 197.1 & 0.01 \\
$1+t+t^{2}+t^{3}$ & -6.1 & -2.5 & 27.5783 & 0.07149 \\
$\ln (1+t)$ & -9.1 & -4 & 2.5925 & 0.7743 \\
\hline
\end{tabular}

TABLE 2: Minimal and maximal logarithmic errors of approximation for various source functions: hyperbolic potential.

\begin{tabular}{lcccc}
\hline$f(t)$ & $\min \operatorname{Er}$ & $\max \operatorname{Er}$ & $a_{0}$ & $s$ \\
\hline$\delta(t)$ & -6.8 & -6.55 & 2 & 1 \\
$\theta(t)$ & -9.1 & -4.25 & 2.1483 & 0.93107 \\
$\sin (t)$ & -9.8 & -5.7 & 2.77245 & 0.72126 \\
$\exp (t)$ & -7.3 & -3.5 & 200.3 & 0.01 \\
$1+t+t^{2}+t^{3}$ & -7.2 & -3.4 & 28.0215 & 0.07149 \\
$\ln (1+t)$ & -9.7 & -5.25 & 2.5824 & 0.7743 \\
\hline
\end{tabular}

exponential, polynomial, and logarithmic influences shows a high efficiency as well (see Figures 2-6).

The minimal and maximal logarithmic errors for the considered source functions are reported in Table 1 . The scale factors $a_{0}$ and $s$ are chosen to minimize the Er function.

4.2. Hyperbolic Potential. Now consider the case when the potential is given by (26). The error analysis for the same source functions as above is reported in Figures 7-12 and Table 2. It is observed that when we use the same values of the scaling factor $s$ for both nonlinearities, then the corresponding values of $a_{0}$ are also close to each other.

\section{Conclusion}

The validity of Frasca's short time expansion (nonlinear Green's representation formula) developed recently for second-order ordinary differential equations of a specific form is established numerically for new classes of nonlinear equations. It is shown that by means of the method of generalized separation of variables, a hierarchy of nonlinear wave equations can be reduced to second-order nonlinear ordinary differential equations, the solution of which can be represented in terms of the nonlinear Green's function.

The numerical solution derived by means of the method of lines is compared with the approximate solution represented in terms of nonlinear Green's function in the case of quadratic and hyperbolic nonlinearities. Consideration of different types of source influences ensures the robustness of the technique. It is observed that the logarithmic error of the two solutions strongly depends on the right-hand side of the equation, corresponding to the source influencing the real-life object or phenomena. The approximation error is minimized with respect to two scaling factors occurring in the right-hand side of the nonlinear equation for Green's function and in the first-order term in Frasca's short time expansion. The approach can be used to derive explicitapproximate solutions to various other nonlinear partial differential equations.

\section{Data Availability}

No data were used to support this study.

\section{Conflicts of Interest}

The author declares that there are no conflicts of interest regarding the publication of this paper.

\section{References}

[1] G. Adomian, Solving Frontier Problems of Physics: The Decomposition Method, Kluwer Academic, Dordrecht, The Netherlands, 1994.

[2] R. Hirota, The Direct Method in Soliton Theory, Cambridge University Press, Cambridge, UK, 2004.

[3] M. Wang, X. Li, and J. Zhang, “The $G^{\prime} / G$-expansion method and travelling wave solutions of nonlinear evolution equations in mathematical physics," Physics Letters A, vol. 372, no. 4, pp. 417-423, 2008.

[4] D. G. Duffy, Green's Functions with Applications, Studies in Advanced Mathematics, Chapman \& Hall/CRC, Boca Raton, FL, USA, 2 edition, 2001.

[5] D. G. Cacuci, R. B. Perez, and V. Protopopescu, "Duals and propagators: a canonical formalism for nonlinear equations," Journal of Mathematical Physics, vol. 29, no. 2, pp. 353-361, 1988.

[6] D. G. Cacuci and V. Protopopescu, "Propagators for nonlinear systems," Journal of Physics A: Mathematical and General, vol. 22, no. 13, pp. 2399-2414, 1989.

[7] D. G. Cacuci and O. A. Karakashian, "Benchmarking the propagator method for nonlinear systems: a Burgers-Kortewegde Vries equation," Journal of Computational Physics, vol. 89, no. 1, pp. 63-79, 1990.

[8] M. Frasca, "Green function method for nonlinear systems," Modern Physics Letters A, vol. 22, no. 18, pp. 1293-1299, 2007.

[9] M. Frasca, "Green functions and nonlinear systems: short time expansion," International Journal of Modern Physics A, vol. 23, no. 2, pp. 299-308, 2008.

[10] As. Zh. Khurshudyan, "New Green's functions for some nonlinear oscillating systems and related PDEs," International Journal of Modern Physics C, vol. 29, no. 4, Article ID 1850032, 2018.

[11] C. Guo, J. Guo, Y. Gao, and S. h. Kang, "Existence of Positive Solutions for Two-Point Boundary Value Problems of Nonlinear Finite Discrete Fractional Differential Equations and Its Application," Advances in Mathematical Physics, 2016.

[12] Z. Gao and J. Wang, "Hyers-Ulam Stability and Existence of Solutions for Nigmatullin's Fractional Diffusion Equation," Advances in Mathematical Physics, vol. 2017, pp. 1-6, 2017.

[13] A. S. Avetisyan and A. Z. Khurshudyan, "Green's function approach in approximate controllability for nonlinear physical processes," Modern Physics Letters A, vol. 32, no. 21, 1730015, 7 pages, 2017.

[14] A. S. Avetisyan and As. Zh. Khurshudyan, Controllability of Dynamic Systems: The Greens Function Approach, Cambridge Scholars Publishing, Cambridge, 2018. 
[15] A. D. Polyanin, A. I. Zhurov, and A. V. Vyaz'min, "Exact solutions of nonlinear heat- and mass-transfer equations," Theoretical Foundations of Chemical Engineering, vol. 34, no. 5, pp. 403-415, 2000.

[16] A. F. Barannyk, T. A. Barannyk, and I. I. Yuryk, "Generalized separation of variables and exact solutions of nonlinear equations," Ukrainian Mathematical Journal, vol. 62, no. 12, pp. 18511865, 2011.

[17] J. D. Murray, Mathematical Biology. Vol. 1: An Introduction ¿amp; Vol. 2: Spatial Models and Biomedical Applications, Springer, Berlin, Germany, 3 edition, 2002.

[18] D. S. Drumheller, Introduction to Wave Propagation in Nonlinear Fluids and Solids, Cambridge University Press, Cambridge, 1998. 


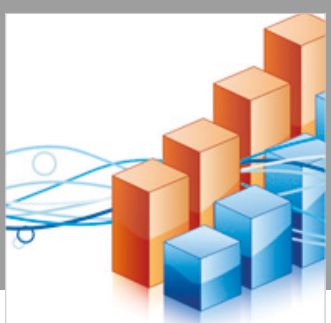

Advances in

Operations Research

\section{-n-m}
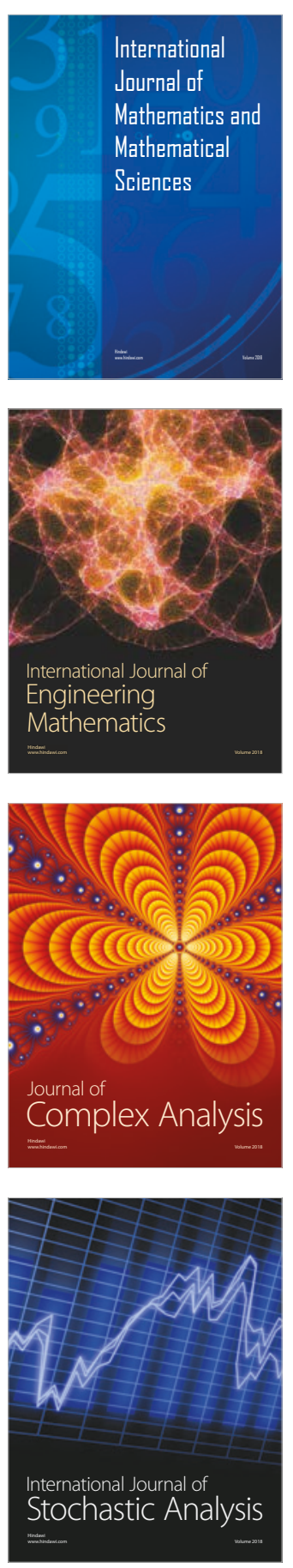
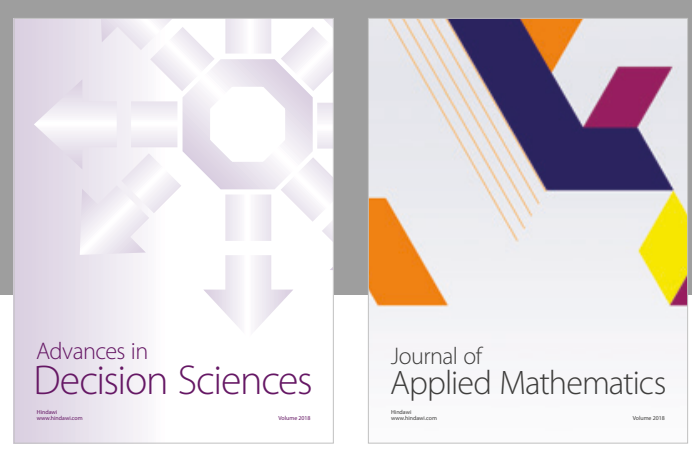

Journal of

Applied Mathematics
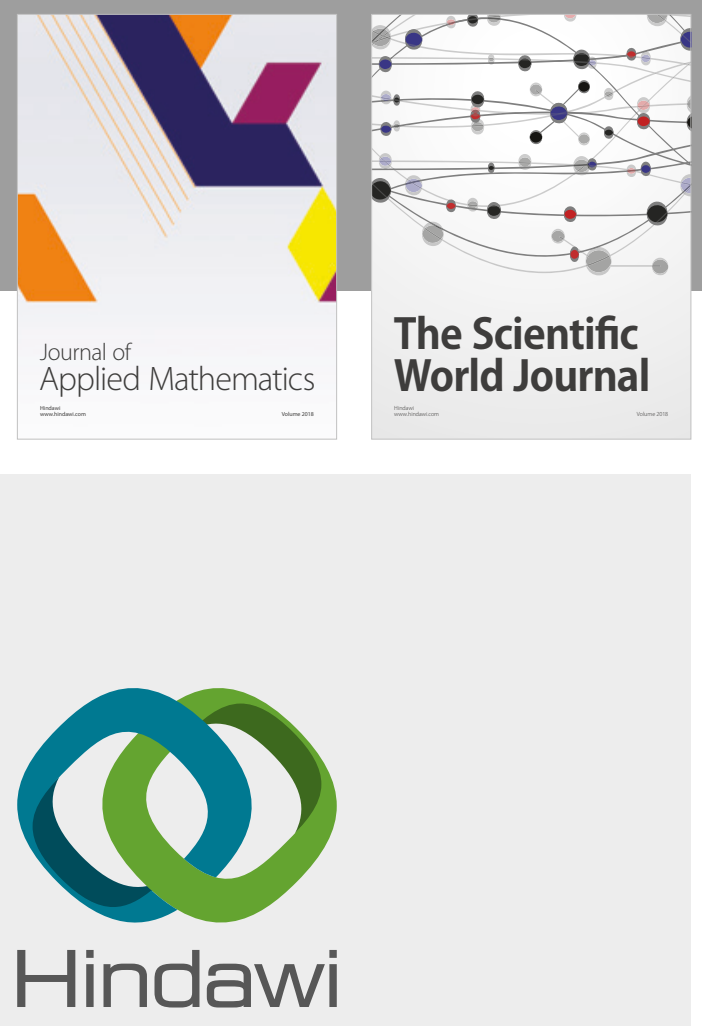

Submit your manuscripts at

www.hindawi.com

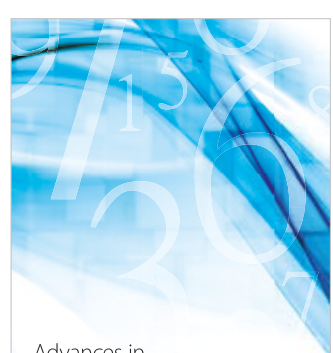

Advances in
Numerical Analysis
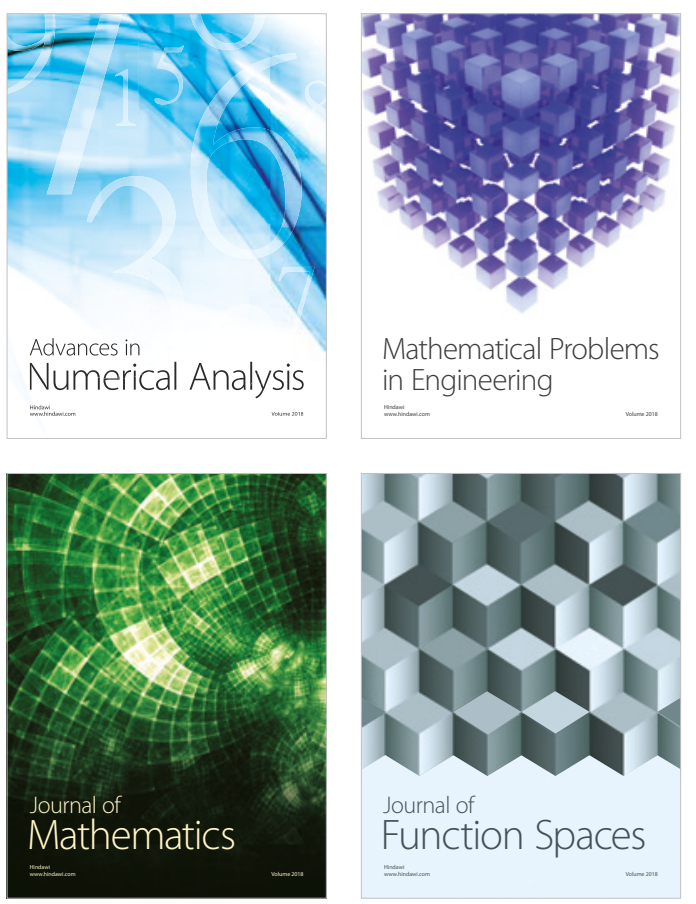

Mathematical Problems in Engineering

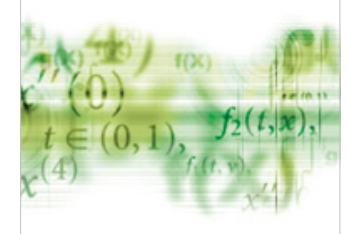

International Journal of

Differential Equations

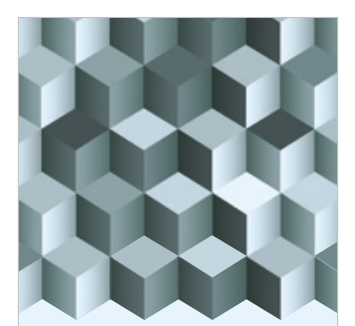

Journal of

Function Spaces

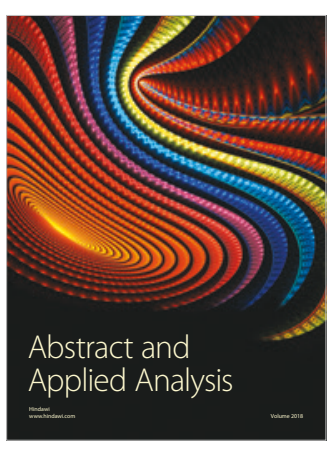

The Scientific

World Journal

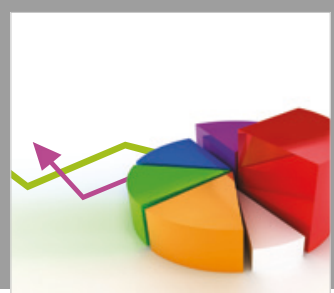

Journal of

Probability and Statistics
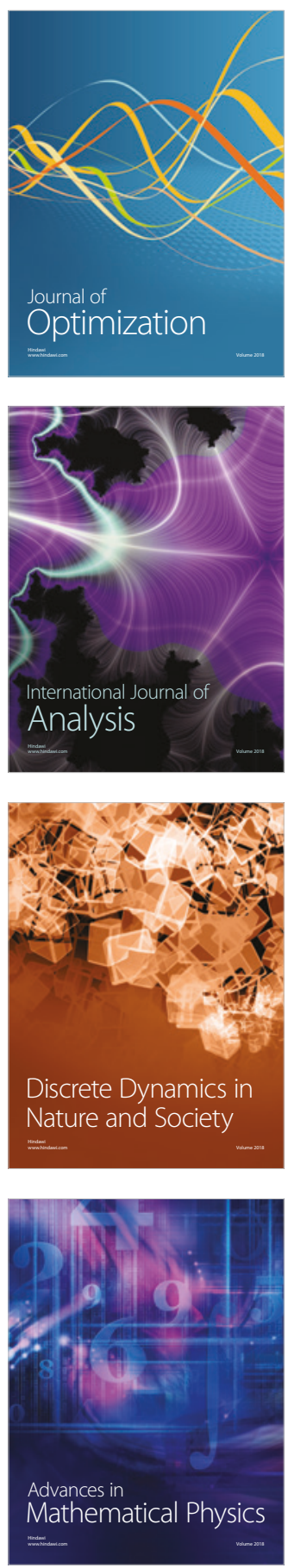